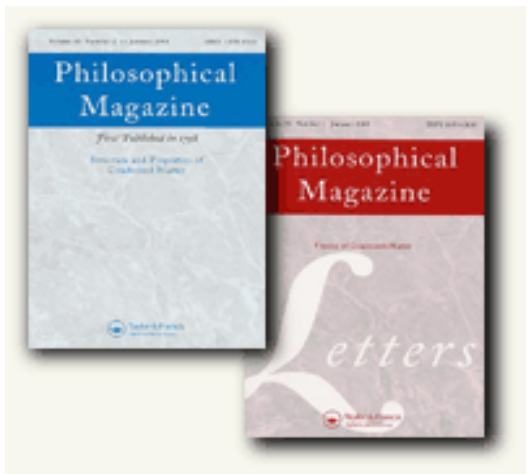

\title{
Mesoscale thermodynamic analysis of atomic-scale dislocation-obstacle interactions simulated by molecular dynamics
}

\begin{tabular}{|r|l|}
\hline Journal: & Philosophical Magazine \& Philosophical Magazine Letters \\
\hline Manuscript ID: & TPHM-09-Apr-0156.R1 \\
\hline Journal Selection: & Philosophical Magazine \\
\hline Date Submitted by the \\
Author: & 14-Jun-2009 \\
\hline Keywords: List of Authors: & $\begin{array}{l}\text { Monnet, Ghiath; EDF, MMC } \\
\text { Osetsky, Yuri; Oak Ridge National Laboratory, Materials Science } \\
\text { and Technology Division } \\
\text { Bacon, David; The University of Liverpool, 2Department of } \\
\text { Engineering }\end{array}$ \\
\hline Keywords (user supplied): & $\begin{array}{l}\text { atomistic simulation, dislocation dynamics, dislocation interactions, } \\
\text { deformation }\end{array}$ \\
\hline & atomistic simulation, dislocation dynamics, dislocation interactions \\
\hline
\end{tabular}

\section{scholarONE" \\ Manuscript Central}




\begin{abstract}
Given the time and length scales in molecular dynamics (MD) simulations of dislocationdefect interactions, quantitative MD results cannot be used directly in larger scale simulations or compared directly with experiment. A method to extract fundamental quantities from MD simulations is proposed here. The first quantity is a critical stress defined to characterise the obstacle resistance. This mesoscopic parameter, rather than the obstacle "strength" designed for a point obstacle, is to be used for an obstacle of finite size. At finite temperature, our analyses of MD simulations allow the activation energy to be determined as a function of temperature. The results confirm the proportionality between activation energy and temperature that is frequently observed by experiment. By coupling the data for the activation energy and the critical stress as functions of temperature, we show how the activation energy can be deduced at a given value of the critical stress.
\end{abstract}

\footnotetext{
${ }^{1}$ Contact details: telephone (+33160736473), fax (+33160736889), e-mail: ghiath.monnet@edf.fr
} 


\section{Introduction}

In a recent work [1], data on the mechanics of dislocation-defect interaction at $0 \mathrm{~K}$ obtained by molecular statics (MS) simulations in [2] were analysed in a mesoscopic framework. It was shown that the change in potential energy as a dislocation encounters and finally cuts through a periodic row of voids can always be expressed as the sum of three different components: (i) the energy of elastic deformation, (ii) the energy associated with the elongation of the dislocation as it bows out between obstacles and (iii) the interaction energy between the dislocation and an individual void. At finite temperature, the response of the system under mechanical load is no longer deterministic, for the local features of the interaction between a dislocation and obstacle introduce local energy fluctuations, even in isolated systems $[3,4]$. These fluctuations assist dislocations to unpin, thereby inducing an irreversible evolution of the whole system. This explains the general decrease of the obstacle strength with increasing temperature.

Although a large number of studies by molecular dynamics (MD) simulations of dislocation-obstacle effects have been reported in the literature, see for example [5,6,7], little has been done to analyse the nature of the localised thermal activation process. For example, recent investigations [8,9], although applied to the Peierls barrier rather than the localized obstacle case, provided two different treatments. Thus, analysis of the motion of a screw dislocation in iron in [8] based on a time-independent rate equation showed that the stress dependence on temperature can be correlated with the time-independent integration of the jump probability. This method provides a self-consistent definition of the critical stress that accounts for its dependency on strain rate and temperature. The properties deduced from this integration provided an explanation of the shift between the critical stress measured in MD simulation and experiment. In the MD simulation of the motion of the Lomer dislocation in aluminium in [9], another approximation is used to deduce the critical stress. The incompatibility of these two approaches is the subject of a forthcoming paper.

In a completely different approach, Jarzynsky [10,11] and Crooks [12] proposed a connection between equilibrium statistical mechanics and systems driven far from equilibrium within conditions of microscopic reversibility. The established theory, however, cannot be usefully applied in the case of thermal barriers.

It should also be noted that interpretation of the critical stress reached in MD simulations is not straightforward. For example, the connection with theory via the line tension approximation is not successful [13] because of the absence of an appropriate 
definition of the interaction strength in the case of finite size obstacles. The classical picture of strength defined by a critical cusp angle on the dislocation line, see for example [14], cannot be used even if the local dislocation character is taken into account (see below) [13].

In this paper, we propose a new approach to analyse dislocation-obstacle interactions at finite temperature. We illustrate it for the case of edge dislocation-void interaction, as in [13]._The paper is organized as follows. First, we describe briefly in section 2 the features of the atomic-scale simulations used here. Then the analysis reported in [1] is applied and extended in section 3 in order to interpret the results of MD simulations. In this section we provide a new definition of the absolute strength of finite-size obstacles that is appropriate for mesoscopic treatment. The MD results of the dislocation-void interaction are presented and the effect of the temperature is discussed in section 4 . The thermodynamic treatment of the interaction process is presented in section 5 .

\section{MS and MD methods for simulating dislocation-obstacle interactions}

The source of the mesoscale analysis introduced in this paper is data from atom-scale modelling of a gliding edge dislocation in $\alpha$-iron that interacts with an obstacle in the form of a spherical void centred on the glide plane. The edge dislocation with the Burgers vector $\boldsymbol{b}=$ $1 / 2[111]$ parallel to the $\mathrm{x}$ axis is initially aligned along the $[11 \overline{2}]$ direction of the $\mathrm{y}$ axis and glides on the $(1 \overline{1} 0)$ plane. We have used the two simulation techniques MS and MD with the interatomic potential developed by Ackland et al. [15], for which the empirical parameters were fitted for BCC iron. The atomic model contained 877,590 lattice sites and its $\mathrm{x}, \mathrm{y}$ and $\mathrm{z}$ dimensions were approximately $25 \mathrm{~nm}(\sim 100 b), 21 \mathrm{~nm}$ and $20 \mathrm{~nm}$, respectively. The void consisted of 59 vacancies and had diameter, $D$, equal to $1 \mathrm{~nm}$. Details of techniques to create the dislocation, visualize its core atoms, induce its motion and calculate stress and strain are presented in [2]. Here we just mention a few details.

In the MS simulations, the dislocation moved due to resolved shear strain, $\gamma$, applied in increments $\Delta \gamma$. The potential energy of the model was minimized by atomic relaxation after each increment, and the potential energy and the applied shear stress, $\tau$, corresponding to the strain were then calculated for that set of atomic coordinates. The atomic positions obtained in this way represent the equilibrium configuration of the dislocation interacting with the void at each value of $\tau$ and $\gamma$, and are therefore the atomic-level equivalent to the dislocation line 

simulations were performed with $\Delta \gamma$ equal to either $10^{-4}$ or $5 \times 10^{-5}$.

Before proceeding, we recall the energy contributions analysed in the original paper [1]. In MS simulations, the incremental applied work $\delta W_{a p p}$ and the change in potential energy $d U_{p o t}$ are coupled through the equation $\delta W_{a p p}=\Delta U_{p o t}+\delta Q$, where $\delta Q$ is the heat extracted from the system in order to keep it at temperature $T=0 \mathrm{~K}$. It accounts for all irreversible processes in the simulation [12], namely work against the lattice friction $W_{p l}$ and the annihilation of a dislocation segment inside the void. The interaction energy $\left(E_{i n t}\right)$ can be deduced from the relation $E_{\text {int }}=U_{\text {pot }}-E_{\text {curv }}-E_{e l}$, where $E_{\text {curv }}$ is the energy necessary to bend the dislocation before it unpins from the void and $E_{e l}$ is the energy of the elastic deformation of the model due to the applied shear stress.

MD simulations were carried out for the temperature range 1 to $600 \mathrm{~K}$ at values of the applied strain rate, $\dot{\gamma}$, equal to $1 \times 10^{6} \mathrm{~s}^{-1}$. Additional simulations for $600 \mathrm{~K}$ were also carried out for $\dot{\gamma}=5 \times 10^{6} \mathrm{~s}^{-1}$. At these strain rates the steady state dislocation velocity is 2.0 and $10.0 \mathrm{~ms}^{-1}$ for the model size used. The total simulation time varied from 1.6 to $16 \mathrm{~ns}$. During this time, the temperature of the model, calculated from the average kinetic energy of all the atoms, rose by less than $1 \mathrm{~K}$. This increase consists of two contributions. One is due to friction in dislocation motion and depends on the dislocation velocity. The dislocation accelerates quickly when attracted into the void and when leaving it at the maximum stress. The other contribution arises from the decrease in potential energy when the dislocation enters the void, losing part of its length, and leaves it, releasing the accumulated elastic energy. The latter is the biggest contribution in the cases considered but occurs after release of the dislocation and does not affect the dislocation-void interaction analysis. The local temperature increase due to potential energy change is an unavoidable yet realistic phenomenon. This thermal energy diffuses over the surrounding atoms but does not homogenize completely within the simulation time. There was practically no change in the temperature near the crystallite boundaries in these simulations and it was unnecessary to apply a thermostat.

For statistical treatment of the results, some simulation conditions were repeated up to five times but with different initial atomic velocity distributions. The principal outputs of the MD simulation for a given temperature are the dependence of applied stress, potential energy and kinetic energy on applied strain and/or time. 


\section{Results of MS simulations}

\subsection{Dependence of stress and energy on applied strain}

Some results of an MS simulation $(T=0 \mathrm{~K})$ are seen in Figure 1, where $\tau$ versus $\gamma$ is plotted in (a) and the potential and other energy changes are plotted against $\gamma$ in (b). The shear strain $\gamma_{r}$ denotes the reference state in which the dislocation is straight and penetrates the void through its centre. It corresponds to $\tau$ equal to the friction stress, $\tau_{f}$, for the edge dislocation, which is close to $23 \mathrm{MPa}$.

Fig. 1 : about here

At this stress level, the potential energy is not minimum, for although the curvature energy is zero, the elastic energy is $V \tau_{f}^{2}, 2 \mu$, where $V$ is the volume of the simulation box and $\mu$ the shear modulus. At $\gamma_{r}, E_{\text {int }}$, is equal to $-7.5 \mathrm{eV}$, compared to the value $-17.4 \mathrm{eV}$ found in the data for a $2 \mathrm{~nm}$ void [1]. The former value is approximately half of the latter, demonstrating that the interaction energy, deduced from the analysis in [1], is a characteristic of the dislocation-obstacle interaction and independent of the simulations conditions. This interaction energy is basically needed to form the two steps on the void surface created when the dislocation enters and shears the void. However, the difference between -7.5 and half of 17.4 is due to the fact that the energy of the surface step on the $1 \mathrm{~nm}$ void is not simply half that of the step on the $2 \mathrm{~nm}$ void.

\subsection{Critical strength of obstacles of finite size}

It is clear that during pinning of the dislocation by the void, mechanical equilibrium prevails around the obstacle. Unpinning occurs when the obstacle experiences an effective force due to the dislocation curvature that is larger than it can sustain. The conventional picture of this equilibrium is that of point obstacles [14,16], depicted in Figure 2a. The line tension (LT), $\Gamma$, on each side of the obstacle generates a resultant force $F=2 \Gamma \cos (\varphi / 2)$, where cusp angle $\varphi$ reflects the obstacle strength. With an increasing line curvature, $\varphi$ decreases and $F$ increases. 
When the force exceeds the resistance of the obstacle, $F_{\max }$, the dislocation unpins from the obstacle.

Fig. 2 : about here

This scheme has been used in theories of alloy strengthening, for a review see [17]. It was also used for the treatment of the Orowan mechanism [18] by employing a line tension that accounts properly for the dipole interaction between dislocation arms around the particle. However, the line tension approximation suffers from three principal limitations. (i) It is not easy to extend it to obstacles of finite size. The critical stress it gives with the actual $\varphi$ for a void is not in good agreement with MD results [13]. (ii) The method implies $\Gamma$ can be defined in the vicinity of the obstacle, but this is uncertain because the LT in the vicinity of the obstacle depends on the orientation of the line [19] and is not the same as that due to line curvature [20]. (iii) The force balance on a dislocation segment is not necessarily parallel to the segment direction [20]. A different method can be used to resolve these issues [21]. In the case of an obstacle in a periodic row, as shown in Figure $2 \mathrm{~b}$, imagine the obstacle to be equivalent to a dislocation segment of length $w$ which experiences a stress preventing its motion. The obstacle experiences a force composed of two components: (a) the force $\tau b w$ resulting from the applied stress $\tau$, where $w$ the shortest distance between dislocation arms touching the obstacle, as defined in Figure $2 b$, and (b) the force resulting from the curvature of the dislocation when the applied stress exceeds the friction stress, $\tau_{f}$, resulting from the lattice friction on the edge dislocation. The second component is given by $l b\left(\tau-\tau_{f}\right)$, where $l$ is the spacing between obstacles shown in Figure $2 \mathrm{~b}$. The following equation is imposed by the condition of force balance:

$$
\tau_{e f f}=\left(1+\frac{l}{w}\right) \tau-\frac{l}{w} \tau_{f},
$$

where $\tau_{\text {eff }}$ is the 'effective' stress experienced by the obstacle when the applied stress is $\tau$. Mechanical equilibrium prevails as long as the obstacle counterbalances $\tau_{\text {eff }}$ with an equal resistance in the opposite direction. At $T=0 \mathrm{~K}$ unpinning proceeds when $\tau_{\text {eff }}$ exceeds the maximum obstacle resistance, $\tau_{c}^{o}$, which can be deduced from Equation (1) when the applied stress reaches its maximum $\tau_{\max }$. 
In most cases, $l$ is much larger than $w$ and this leads to the following approximation:

$$
\tau_{c}^{o}=\frac{l}{w}\left(\tau_{\max }-\tau_{f}\right) .
$$

It should be noted that Equation (2) is valid as long as the friction stress is independent of the dislocation character. Therefore, this method cannot be applied when a screw dislocation segment is formed during the interaction, which is the case for large void size [5], because of the large Peierls stress known to exist for the screw dislocation in iron at room temperature and below [8]. The idea of connecting unpinning to a critical effective stress rather than a critical force was already used successfully in dislocation dynamics simulations to predict strengthening by different mechanisms [22,23]. Here, we extend the method with the help of MD simulations to characterise void strength.

In the case of voids, the effective stress is associated with the stress felt by atoms on the void surface on both sides of the slip plane, tending to create the missing dislocation segment of length $w$ that has been annihilated inside the void. The other term, $\tau_{c}^{o}$, can be interpreted as the maximum resistance of the lattice to the creation of an edge dislocation at a curved free surface. $\tau_{c}^{o}$ should then be a characteristic material property and must be independent of the void size and simulation conditions. This point can be checked using Equation (2) if the critical shape of the dislocation is known for different void sizes and dislocation lengths.

An MS study for different void sizes was reported in [5]. In order to check the validity of our method, we select the cases of relatively weak obstacles, i.e. $D=0.9,1,1.5$ and $2 \mathrm{~nm}$, for which unpinning is controlled by dislocation curvature. Data for larger voids cannot be used because MS simulations show that straight screw dipoles are generated at the obstacle before unpinning, which connects the unpinning process to the mobility of screw segments. For the different sizes considered, the values of $w$ are taken to be equal to $4 a \sqrt{6} / 3$, $5 a \sqrt{6} / 3$ and $6 a \sqrt{6} / 3$, where $a$ is the lattice parameter. These values correspond to the integer number of interplanar spacings along $[11 \overline{2}]$ that are closest to the observed critical width. According to Equation 2, a plot of $\tau_{\max }$ versus $w / l$ should give a straight line with intercept $\tau_{f}$ (approx. $23 \mathrm{MPa}$ ) on the $\tau_{\max }$ axis. This is tested in Figure 3, where MS results for 
$\tau_{\max }$ obtained in [5] with simulation boxes with length along the $\mathrm{y}$ axis equal to either 42 or $83 \mathrm{~nm}$ are plotted as circles or rectangles, respectively.

Fig. 3 : about here

The error bar for $w / l$ is estimated from the uncertainty in determining $w$ due to width of the dislocation core in visualization of core atoms. The dashed line represents the best linear fit passing through the point $\left(0, \tau_{f}\right)$. It is seen that the MS data are well aligned with the dashed line. The maximum obstacle resistance, $\tau_{c}^{o}$, given by the gradient of the line is constant (4230 MPa) and therefore characteristic of the strength of voids, independent of $D$ and $L$. It will be seen that the value $\tau_{c}^{o}=4230 \mathrm{MPa}$ is important for our treatment.

\section{MD simulations results}

Thermodynamic analysis at finite temperature is complex since thermal activation introduces a time-dependence into the interaction process and quasi-static treatment is no longer valid. However, the first law of thermodynamics for energy conservation is still valid, but with the difference that the heat generated is not extracted from the system. This leads to an increase in temperature, $\Delta T$, of the system.

The major contribution to this heat is dissipated work due to lattice friction [1]. It increases with decreasing temperature. The maximum value corresponds to the heat extracted from the system in MS simulation. The corresponding change in $\Delta T$ would be given by

$$
\Delta T=\frac{1}{C_{v}}\left[V \int_{0}^{\gamma_{t}} \tau d \gamma-\Delta U_{p o t}\right],
$$

where $C_{v}$ is a heat capacity at constant volume, $d \gamma$ the increment of applied strain, $\gamma_{t}$ the total shear strain and $\Delta U_{p o t}$ the difference in the potential energy between the beginning and end of the simulation. The term in brackets in Equation (3) is the difference between the total mechanical energy provided to the system and the change in the potential energy, which corresponds exactly to the extracted heat. This heat amounts to $7.8 \mathrm{eV}$, which corresponds to $\Delta T<1 \mathrm{~K}$ at the end of the simulation. Since this heat is extracted, the transformation is 
Several effects can be distinguished.

(i) $\tau_{f 2}$ which is $23 \mathrm{MPa}$ at $T=0 \mathrm{~K}$, decreases rapidly with increasing $T$, thereby confirming that the dissipated heat can be completely neglected.

(ii) $\tau_{\max }$ decreases with increasing $T$, which is the signature of thermal activation.

(iii) The pinning time decreases with increasing $T$.

(iv) The behaviour of the system is markedly stochastic, since the response is not identical in the independent simulations carried out at the same temperature.

Thus, unpinning occurs at different stress levels and different loading times. The latter is a specific feature of thermal activation. The amount of mechanical work necessary for unpinning is not the same in the different simulations and so a rigorous analysis should account for this. It will be seen in the following that the temperature effect and thermal activation can be analysed only on the mesoscopic scale. By 'mesoscopic' we mean the scale on which the atomic nature of the material is smoothed and replaced by other contiunuum models such as elasticity, elasto-plasticity, etc. For example, it is typically the scale used in dislocation dynamics simulations.

\section{Thermodynamic analyses}

\subsection{Free energy and the reaction coordinate}


At the mesoscopic scale, the atomic-level parameters should be replaced by homogenous variables. As the applied stress increases during straining from the reference strain $\gamma=\gamma_{r}$, effective stress given by Equation (1) increases. This leads to relative displacement of atoms above and below the slip plane at the void surface, generating a local shear. A convenient way to describe the progress of the interaction is to introduce a scalar variable, $\xi$, representing the interaction coordinate, see Figure 5. However, it is difficult to assign a precise physical meaning to $\xi$ in our case since atomic displacements are not restricted to the atomic rows belonging to the void surface.

Fig. 5 : about here

Following the discussion above, the transformation can be considered isothermal at constant pressure. The system potential is thus the Gibbs free energy, $G$, [24] and not the enthalpy. Figure 5 is a scheme of the interaction giving the evolution of the change $\Delta G\left(\tau_{\text {eff }}=\right.$ $0)$ in $G$ as a function of $\xi$ in the absence of stress. Initially the system is in its ground state labelled I. To overcome the whole barrier, the free energy $\Delta G_{\max }$ should be provided in order to bring the system reversibly to its saddle state B. The derivative of the free Gibbs energy with respect to $\xi$ gives the resistance stress $\tau_{\text {lattice }}$, i.e. $\partial \Delta G\left(\tau_{\text {eff }}=0\right) / \partial \xi=b w \tau_{\text {lattice. }}$ A schematic representation of $\tau_{\text {lattice }}$ is depicted in Figure 5. Under the effect of an applied effective stress $\tau_{\text {eff, }}$, the system potential $\Delta G\left(\tau_{\text {eff }}\right)$ evolves due to the work done by the effective stress, see Figure 5, and is now characterised by a new ground state labelled A at $\xi$ where $\tau_{\text {latice }}=-\tau_{\text {eff. }}$ Also, the saddle state becomes $\mathrm{S}$ and the energy barrier decreases to $\Delta G\left(\tau_{\text {eff }}\right)$ because of the work done by $\tau_{\text {eff }}$ during the transition from state $\mathrm{A}$ to state $\mathrm{S}$. The system would stay at state $\mathrm{A}$ forever at $0 \mathrm{~K}$. The unpinning of the dislocation occurs only when the effective stress is increased to its critical maximum value $\tau_{c}^{o}$. At a temperature greater than $0 \mathrm{~K}$, there is a non-zero probability for the missing free energy $\Delta G\left(\tau_{\text {eff }}\right)$ to be supplied by the rest of the crystal, leading to the release of the dislocation.

It is important to note that the free energy profile and the corresponding reasoning are valid for quasi-static progress of the reaction. This, of course, is not the case during the thermal activation event, when the transition occurs in a time interval of the same order of magnitude as the period of atomic vibration [25]. The reaction coordinate has to be reconsidered correspondingly as a function of the inertial effects, which, to our knowledge, is 
not covered by any theoretical or simulation investigation reported in the literature. This is the main problem of all approaches tending to estimate the activation energy directly from the Gibbs free energy profile $[9,26,27]$. The approach proposed in this paper for determination of the activation energy allows this difficulty to be avoided.

\subsection{Connection with statistical dynamics}

Let us restrict our system to a small volume of atoms $\Omega$ surrounding the void. This volume is in thermal equilibrium with the rest of the crystal at temperature $T$. Each MD simulation can be considered as a sample path from an initial state $\mathrm{I}$ at time $t=0$ to the saddle state $\mathrm{S}$ at $t=$ $\Delta t$, see Figure 5. An appropriate choice of these states facilitates the theoretical treatment. In our case, the natural choice for the initial and final states corresponds to the reference state $\gamma_{r}$ and the saddle state, respectively. The latter corresponds to the state of the maximum Gibbs free energy. Within every such path, work $W$ is done by the effective stress on the volume $\Omega$. According to Jarzynsky [10,11] and Crooks [12], under the condition of microscopic reversibility, the increase of the system potential can be obtained by averaging the work done on the system over all sample paths:

$$
\Delta G_{\max }=G_{S}-G_{I}=-\frac{1}{\beta} \ln \langle\exp (-\beta W)\rangle
$$

where $\beta$ is the temperature parameter $(k T)^{-1}$ and $k$ is the Boltzmann constant. The brackets $<>$ in Equation (4) denote the average over all paths. The average change in the system potential is, in general, less than the entire work done on the system, because of dissipation. But Equation (4) stipulates that the modification of the system potential is a simple function of the average over all possible paths of the work done on the system. In our special case, all paths are characterised by the same initial and final states. The difference between $G_{S}$ and $G_{I}$ is therefore exactly equal to the height of the energy barrier $\Delta G_{\max }$, see Figure 5 .

Among the different paths in Equation (4), some of the statistical systems acquire the additional quantity of energy corresponding to the missing energy, $\Delta G=\Delta G_{\max }-W$, due to thermal fluctuations and therefore reach the saddle state. Once a system acquires $\Delta G$ from the surrounding crystal, it undergoes an irreversible transformation beyond the saddle state B. This is why all paths that can be computed through MD simulations are restricted to only 
paths in which the system has acquired the activation energy. In contrast, the average in Equation (4) covers all possible paths and not only paths selected in MD simulations. This is the reason behind the difference between the expected change in energy $\Delta G \equiv W$ and the acquired value $\Delta G_{\max }$.

Following the dynamical approach and equilibrium statistics [28], the probability of acquiring an additional energy $\Delta G$ is proportional to the Boltzmann factor $\exp (-\beta \Delta G)$. The exact jump rate $\omega[4]$ can then be given by $\omega=v \exp [-\beta \Delta G]$, where $v$ is a frequency parameter to be discussed later. This rate equation stipulates that the probability of unpinning depends only on the instantaneous chance to receive $\Delta G$ from the rest of the crystal. During a time increment $d t$, the infinitesimal probability of acquiring $\Delta G$ is:

$$
d p=v \exp (-\beta \Delta G) d t
$$

The probability $p$, increasing with time, reflects the chance that every one of the statistical systems passes through the activated stress.

\subsection{Activation energy under constant stress conditions}

We investigate here the unpinning process in the simple condition of constant effective stress, $\tau_{\text {eff }}=\tau_{c}$. Since $\tau_{c}$ is lower than $\tau_{c}^{o}$, the system is in a stable equilibrium state called the ground state A in Figure 5. The system $\Omega$ is in thermal equilibrium with the rest of the crystal at finite temperature $T$. We assume that at $t=0$ the volume $\Omega$ is in its ground state A, characterised by a given value of the thermodynamic potential. The dislocation unpins when thermal fluctuations provide the missing mechanical energy $\Delta G$. Here we should note that (i) the energy barrier is assumed to be solely a function of stress and (ii) $\Delta G$ is a function of the effective stress defined in Equation (1), and not of the applied stress. This point is important since most treatments reported in the literature assume a dependency on the applied stress (for a review see $[25,29])$. Considering $\Delta G$ to have a dependency on the applied stress is not exact in the case of localised obstacles. It leads to unrealistic values for the activation volume, as will be seen later.

Since $\tau_{e f f}$ is constant, there is no reason for the Boltzmann factor not to be uniformly distributed over time. According to the transition state theory and dynamical theory [25,28], 
the average survival time, or the average time for the system to stay in the half space containing the ground state $\mathrm{A}$, is simply the inverse, $\omega^{-1}$, of the reaction rate. This survival time can be considered as the average incubation time of the unpinning process:

$$
<\Delta t>=v^{-1} \exp \left[\beta \Delta G\left(\tau_{c}\right)\right]
$$

Values of $\Delta t$ could be measured from MD simulations performed at constant applied stress and the Boltzmann factor, exp $\left[-\beta \Delta G\left(\tau_{c}\right)\right]$, then deduced from the average $\langle\Delta t>$ using Equation (6). However, the simulations in the present work used constant applied strain rate loading and required the following treatment.

\subsection{Definition of the critical stress in MD simulation performed at constant strain rate}

For simulations performed under conditions of fixed applied strain rate, a definition has to be assigned to the critical stress $\tau_{c}$ since the applied stress, and consequently the effective stress,

varies with time. When $\tau_{e f f}$ is not constant, Equation (5) is only valid during an infinitesimal time increment $d t$. Here we have to distinguish between the maximum of the effective stress, $\tau_{\max }$, related to the maximum of the applied stress through Equation (2), and the critical stress $\tau_{c}$, characteristic of the unpinning process. This issue was first addressed in [8], where a definition of $\tau_{c}$ was provided. In MS simulations, the critical stress is equal to the maximum stress because the process is quasi-static, but at finite temperature the thermally-activated unpinning depends on time, which precludes the critical stress from being considered equal to the maximum stress. During loading from $\gamma_{r}$ in MD simulations, the effective stress increases with time, which leads to a rapid increase in the accumulated jump probability. The unpinning occurs after an incubation time $\Delta t$. The critical stress is by definition the constant effective stress that would have provided the same jump probability during the same incubation time $\Delta t$. This leads to the following expression:

$$
\Delta t \exp \left[-\beta \Delta G\left(\tau_{c}\right)\right]=\int_{0}^{\Delta t} \exp \left(-\beta \Delta G\left(\tau_{e f f}\right)\right) d t
$$


in which $\tau_{e f f}$ tends to $\tau_{\max }$ when $t$ tends to $\Delta t$. Since the Boltzmann factor is a monotonic increasing function of $\tau_{e f f}$, it turns out from Equation (7) that $\tau_{c}$ is always less than $\tau_{\max }$. The difference between the two quantities depends on the temperature and the activation volume, which accounts for stress sensitivity of activation energy. In order to make progress in estimating the critical stress, we have to postulate a functional form of the activation energy. As a first approximation, we can develop $\Delta G$ in the vicinity of $\tau_{\max }$ by $\Delta G=C-V^{*} \tau_{e f f}$, where $C$ is constant (not to be confused with the value of $\Delta G$ at zero stress) and $V^{*}$ the activation volume. It is important to underline here that our concept of the activation volume does not correspond to the apparent activation volume measured in experiment, $V_{a p p}$. The connection with our definition would be $V^{*}=V_{a p p} \underline{x}(w / l)$. Equation (7) allows us to deduce the critical stress:

$$
\tau_{c}=\frac{1}{\beta V^{*}} \ln \left\langle\exp \left(\beta V^{*} \tau_{e f f}\right)\right\rangle_{\Delta t}
$$

where the integral in Equation (7) is replaced by its average value over the incubation time. The variation of $V^{*}$ in Equation ( $\left.\underline{8}\right)$ is relatively weak compared to the variation of $\tau_{e f f}$. This allows us to deduce $\tau_{c}$ using a rough estimate of $V^{*}$. In our case, it can be set equal to $w b^{2}$, which accounts for the interaction range of the process.

In order to illustrate the difference between $\tau_{\max }$ and $\tau_{c}$, consider the case where the effective stress increases linearly with time from 0 to $\tau_{\max }$. The average in Equation ( $\underline{8}$ ) can then be derived analytically:

$$
\left\langle\exp \left(\beta V^{*} \tau_{\text {eff }}\right)\right\rangle=\frac{1}{\tau_{\max } \beta V^{*}}\left[\exp \left(\beta V^{*} \tau_{\max }\right)-1\right]
$$

It is easy to check that for typical values of $\beta, V^{*}$ and $\tau_{\max }$, the exponential term in Equation (9) is dominant. The critical stress is then given by

$$
\tau_{c}=\tau_{\max }-\frac{k T}{V^{*}} \ln \frac{V^{*} \tau_{\max }}{k T}
$$


Equation (10) confirms that $\tau_{c}$ is less then $\tau_{\max }$ and shows clearly that the difference between them increases with temperature. On the other hand, when the temperature tends to zero, $\tau_{c}$ tends to $\tau_{\max }$. This asymptotic behaviour is expected since, in the absence of thermal activation, the unpinning proceeds only when the effective stress reaches the maximum stress.

Here we have to underline another difficulty in estimating the critical stress. In Equation ( 8$), \tau_{c}$ provided by an MD simulation is only an estimate of the statistically representative critical stress. By repeating the MD simulation at the same strain rate and temperature, we obtain different estimates of $\tau_{c}$. However, averaging should be carried out in the probability space and not in the stress space because the stochastic feature of the activation has its origin in the probability of occupying a given state in the phase space. This probability is proportional to the Boltzmann factor, imposing an exponential dependency on the stress. The arithmetic (linear) average in the stress space is therefore not justified [9]. As a result, the value of $\tau_{c}$ at a given strain rate and given temperature can be calculated from

$$
\tau_{c}=\frac{1}{\beta V^{*}} \ln \left\langle\left\langle\exp \left(\beta V^{*} \tau_{e f f}\right)\right\rangle_{\Delta t}\right\rangle_{n},
$$

where the inner average is to be carried out over time during every MD simulation, while the outer average is to be taken over the number $n$ of repeated MD simulations. The validity of this approach is conditioned by the fact that the repeated MD simulations are independent. The formula in Equation (11) is the same as that developed in [8]. The validity of the treatment in the latter work is thus established under an implicit condition: there is no correlation between the successive kink-pair nucleation events.

The treatment above has been used to calculate the effective stress in our simulations. The results are shown in Figure 6, where it is seen how the maximum and the critical effective stress vary with temperature. However, in order to show the spread of values of $\tau_{c}$, we have used Equation ( $\underline{8})$ rather than Equation (11) and the different values obtained at each temperature are shown in the figure. It can be seen that the spread of values of $\tau_{c}$ at a given temperature is quite small, so the convergence of the different estimates of $\tau_{c}$ is sufficiently fast for our system.

Fig. 6 : about here 
As expected, the difference between the stresses $\tau_{\max }$ and $\tau_{c}$ tends to zero as $T$ tends to $0 \mathrm{~K}$. The difference increases rapidly with increasing $T$ at low temperature, reaching an almost constant value of $0.7 \mathrm{GPa}$ in the range 100 to $600 \mathrm{~K}$. There are two reasons explaining this constant shift at high temperature. As can be seen in Eq. (11), the efficiency of the thermal activation is proportional to $\ln \left(\tau_{\max }\right)$. Also the slope of the function $(k T) \ln (k T)$ decreases strongly with $k T$. It is thus easy to understand why the difference between $\tau_{\text {max }}$ and $\tau_{c}$ should saturate with increasing temperature. This difference, however, amounts to 20 to $25 \%$ of $\tau_{c}$. Consequently, it cannot be neglected and $\tau_{c}$ should not be confused with $\tau_{\max }$ [9]. In other words, the unpinning process is not only determined by the maximum stress, but depends also on the time spent to reach this stress.

\subsection{Calculation of the activation energy}

The aim of this section is to provide a method for estimating the activation energy as a function of the critical stress. The unpinning process depends on three factors, namely the effective stress, the temperature and the deformation rate (through the incubation time), but only two are independent. Equation (ㅁ) shows how these parameters are connected. Since in MD simulations the temperature is almost conserved and $\langle\Delta t\rangle$ can be estimated from different independent simulations, it is possible, in principle, to evaluate the activation energy using Equation (6), provided that the frequency parameter $v$ is known. This question has been addressed several times in the literature, for a review see [4,25]. The effect of the kinetic energy on the activation rate can be considered through the change in the vibrational free energy of the atomic row between state A and S [30]. The determination of $v$ for localised obstacles is complex and involves different characteristics [30]: strength, size, spread, etc. It also involves the vibrational spectrum of the bulk material. Large difference in the estimations of $v$ can be found in the investigations reported in the literature.

However, since the frequency parameter appears as a pre-exponential in Equation (6), only an order of magnitude estimate for its value is needed for the determination of the reaction rate. The commonly-accepted way of doing this is to scale the Debye frequency, $v_{D}$, by the length of the vibrating atomic row [31]. The frequency $v$ can be represented as the frequency of the transverse wave of length equal to that of the effective obstacle, which, in our case, is clearly equal to $w$, the smallest distance at which the dislocation is kept pinned, 
see Figure 2. Since the result of activation is formation of the missing dislocation segment, the vibration frequency of an atomic row of length $w$ should be close to that of a dislocation segment of length $w$. By this procedure, we have $v \approx v_{D} b / w$. However, the vibrating atomic row belongs to the void surface, where the frequency spectrum is lower than that in the bulk [32], and the frequency should be corrected accordingly. As a first approximation, the slowing of atoms in the neighbourhood of the surface can be accounted for by assuming only half of the bulk frequency. For the situation where the stress is constant, Equation (6) can then be rewritten to estimate the activation energy:

$$
\Delta G\left(\tau_{c}\right)=\frac{1}{\beta} \ln \left(<\Delta t>\frac{v_{D} b}{2 w}\right)
$$

But for simulations at constant strain rate, the stress varies from $\tau_{f}$ to $\tau_{\max }$ during loading. This is why the concept of the critical stress was needed in section 5.4 to provide the same jump probability as that measured during constant stress simulations. Equation (12) should thus be coupled to Equation (11). In other words, for every set of simulations performed at a given temperature, we have to evaluate independently the critical stress using Equation (11) and the activation energy using Equation (12). We then construct the two functions $\tau_{c}(T)$ and $\Delta G(T)$ in order to deduce the desired function $\Delta G\left(\tau_{c}\right)$.

The variation of $\Delta G$ with $T$ obtained using Equation (12) is shown in Figure 7. The linear proportionality frequently observed in experiments on BCC and HCP metals such as iron [33, 34], zirconium [35] and titanium [36] is clearly confirmed in our results. This provides evidence for the validity of the two hypotheses that (i) the effect of stress and temperature on the attempt frequency is negligible and (ii) the profile of the energy barrier, i.e. the mechanical work necessary to overcome the barrier without the help of thermal activation, is independent of temperature. The linearity can be described by the relation $\Delta G=$ $C k T$, where $C$ is a constant close to 8.1 .

Fig. 7 : about here

It is also clear that, as with the critical stress, there is no dispersion in the values of the activation energy computed from the independent MD simulations performed for the same temperature. However, two sets of points are clearly distinguishable for $T=600 \mathrm{~K}$. One is 
consistent with the other temperatures, i.e. $C=8.1$, the other is $19 \%$ lower with $C=6.6$. This stems from the fact that the two sets of simulations were performed with different strain rates. The values $C=8.1$ and 6.6 correspond to $\dot{\gamma}=10^{6}$ and $5 \times 10^{6} \mathrm{~s}^{-1}$, respectively. The change in activation energy confirms our assumptions. Increasing the strain rate decreases $\Delta G$ because a faster increase of the applied stress leads to a decrease in the incubation time.

Combining data from Figure 6 for $\tau_{c}$ with that for $\Delta G\left(\tau_{c}\right)$ from Figure 7 enables us to determine the dependence of the activation energy on the critical stress, as shown in Figure 8. Over most of the range, the dependence is almost linear and consistent with a constant value of the activation volume via the relation $\Delta G\left(\tau_{c}\right) \propto-V^{*} \tau_{c}$, which was used to deduce Equation (6). The data give $V^{*}=3.6 b^{3}$, which is close to our estimate made for the evaluation the critical stress. This ensures that the procedure for the estimation of the critical stress is compatible with that used for the evaluation of the activation energy. Beyond the linear region of the plot in Figure 8, $\Delta G\left(\tau_{c}\right)$ tends to zero when $\tau_{c}$ approaches the value found for $T=0 \mathrm{~K}$.

Fig. 8 : about here

The values of the activation energy presented above were measured for critical stresses larger than $2.5 \mathrm{GPa}$. The activation volume does not appear to increase as rapidly as expected when the critical stress falls below this value and tends to zero. Creation of the missing dislocation segment at the void surface should be accompanied, in principle, by a long-range perturbation of the lattice. Although the corresponding mechanical work would still be finite, the activation energy should increase rapidly for low stress [4]. In order to investigate the low stress regime, MD simulations should be carried out at higher temperature and/or much lower strain rates. Unfortunately, this is not feasible with current computer resources.

\section{Conclusions}

It has been shown that a criterion for the obstacle strength of voids, expressed in terms of a critical local effective stress, can be derived from molecular statics calculations carried out to simulate a crystal at temperature $T=0 \mathrm{~K}$. The critical effective stress was found to be independent of the void size, the dislocation length and other simulation parameters. The efficiency of thermal activation in decreasing the critical effective stress is found to be a 


\begin{abstract}
function of the strain rate and the temperature, via the dislocation-void interaction time and the Boltzmann factor, respectively. At a given temperature and strain rate, the activation energy is a function of the effective stress alone. Determination of this activation energy is only possible when the critical stress is defined in the sense that it provides the same unpinning probability during the interaction time as the probability integrated oven the measured stress-strain curve.
\end{abstract}

\title{
Acknowledgments
}

This work was partially supported by the Division of Materials Sciences and Engineering and the Office of Basic Energy Sciences, U.S. Department of Energy, under contract with UTBattelle, LLC. It was also supported by the European project PERFECT ( FI60- CT- 2003208840 and by grant GR/S81162/01 from the UK Engineering and Physical Sciences Research Council. 
References

[1] G. Monnet, Acta Mater. 55 ( 2007) p. 5081.

[2] Yu.N. Osetsky and D.J. Bacon, Model. Simul. Mater. Sci. Eng. 11 (2003) p. 427.

[3] F. Reif, Fundamentals of Statistical and Thermal Physics, Waveland Pr Inc., 1967.

[4] U.F. Kocks, A.S. Argon and M.F. Ashby, Prog. Mater. Sci, vol. 19, Pergamon Press, 1975.

[5] D.J. Bacon, Yu.N. Osetsky and D. Rodney, Dislocations in Solids, eds. J.P. Hirth and L. Kubin, Elsevier, Amsterdam, vol. 15 (2009), in the press.

[6] J. Marian, W. Cai, and V. Bulatov, Nature Mater. 3 (2004) p. 158.

[7] K. Tapasa, Yu.N. Osetsky and D.J. Bacon. Acta Mater. 55 (2007) p. 93.

[8] C. Domain and G. Monnet, Phys. Rev. Lett. 95 (2005) p. 215506.

[9] D. Rodney, Phys. Rev B 76 (2007) p. 144108.

[10] C. Jarzynski, Phys. Rev. Lett. 78 (1997) p. 2690.

[11] C. Jarzynski, Phys. Rev. E 56 (1997) p. 5018.

[12] G.E. Crooks, J. Stat. Phys. 90 (1998) p. 1481.

[13] Yu.N. Osetsky, D.J. Bacon and V. Mohles, Phil. Mag. 83 (2003) p. 3623.

[14] J. Friedel, Dislocations, Pergamon Press, London (1964), p. 31

[15] G.J. Ackland, D.J. Bacon, A.F. Calder and T. Harry, Phil. Mag. A 75 (1997) p. 713.

[16] J.P. Hirth and J. Lothe. Theory of Dislocations, Krieger Publishing Company, 1992.

[17] E. Nembach, Particle Strengthening of Metals and Alloys, Wiley, New York (1997).

[18] D.J. Bacon, U.F. Kocks and R.O. Scattergood, Phil. Mag. 28 (1973) p. 1241.

[19] G. De Wit and J.S. Koehler, Phys. Rev. 116 (1959) p. 1113.

[20] P.M. Hazzledine, H.P. Karnthaler and A. Korner, Phys. Stat. Sol. A 81 (1984) p. 473.

[21] G. Monnet, Phil. Mag. 86 (2006) p. 5927.

[22] V. Mohles, D. Ronnpagel and E. Nembach, Computational Mater. Sci. 16(1999) p. 144.

[23] V. Mohles, Mat. Sci. Eng. A319- 321 (2001) p. 201

[24] G. Schoeck, Phys. Stat. Sol. 8 (1965) p. 499.

[25] G. Schoeck, Dislocation in Solids, Vol. 3, North-Holland Publishing Company, 1980.

[26] A. Seeger P. Schiller, Acta Metall. 10 (1962) p. 348.

[27] J.E. Dorn and S. Rajnak, Metall. Soc. AIME, 230 (1964) p. 1052.

[28] G.H. Vineyard, J. Phys. Chem. Solids 3 (1957) p. 121.

[29] G. Taylor, Progress in Materials Science, 36 (1992) p. 29. 
[30] A.V. Granato, K. Lucke, J. Schlipf and LJ. Teutonico, J. Appl. Phys. 35 (1964) p. 2732.

[31] M. Tang, L.P. Kubin and G.R. Canova, Acta Mater. 46 (1998) p. 3221.

[32] P. Müller and S.Andrieu, Les surfaces solides : concepts et méthodes, EDP Sciences (2005).

[33] W.A. Spitzig, Mater. Sci. Eng. 12 (1973) p. 191.

[34] F.A. Smidt, Acta Metall. 17 (1969) p. 381.

[35] D. Mills and G.B. Craig, Trans. Metal. Soc. AIME 242 (1968) p. 1881.

[36] S. Naka, A. Lasalmonie, P. Costa and L.P. Kubin, Phil. Mag. A 57 (1988) p. 717. 


\section{Figure captions}

Figure 1. Data from an MS simulation. (a) the shear stress $\tau$ versus the shear strain $\gamma$ and (b) change of the potential, elastic, curvature and interaction energies versus $\gamma$.

Figure 2. (a) Conventional picture of mechanical equilibrium for a point obstacle in the line tension approximation. (b) Equilibrium around a finite size obstacle.

Figure $\underline{3}$. The maximum applied stress as a function the ratio $w / l$ for voids (see text for more detail). The dashed line is the best linear fit.

Figure $\underline{4}$. Stress-strain plots for (a) six temperatures and (b) four simulations for $T=300 \mathrm{~K}$. $\dot{\gamma}=10^{6} \mathrm{~s}^{-1}$ in all cases.

Figure $\underline{5}$. Schematic plots of interaction profiles as a function of the interaction coordinate, $\xi$. They represent the change of system energy at zero effective stress, the system energy at a given effective stress and the effective stress needed to push the interaction to a given $\xi$. We simplified the plots by considering the friction stress to be zero.

Figure $\underline{6}$. Dependence of the maximum stress, $\tau_{\max }$, and the critical effective stress, $\tau_{c}$, on temperature for all MD simulations. The values of $\tau_{c}$ were calculated from Equation (9).

Figure 7. Variation of $\Delta G$ with $T$ obtained using Equation (13).

Figure $\underline{8}$. Variation of the activation energy as a function of the critical stress, $\tau_{c}$, obtained from Figures 6 and 7. 

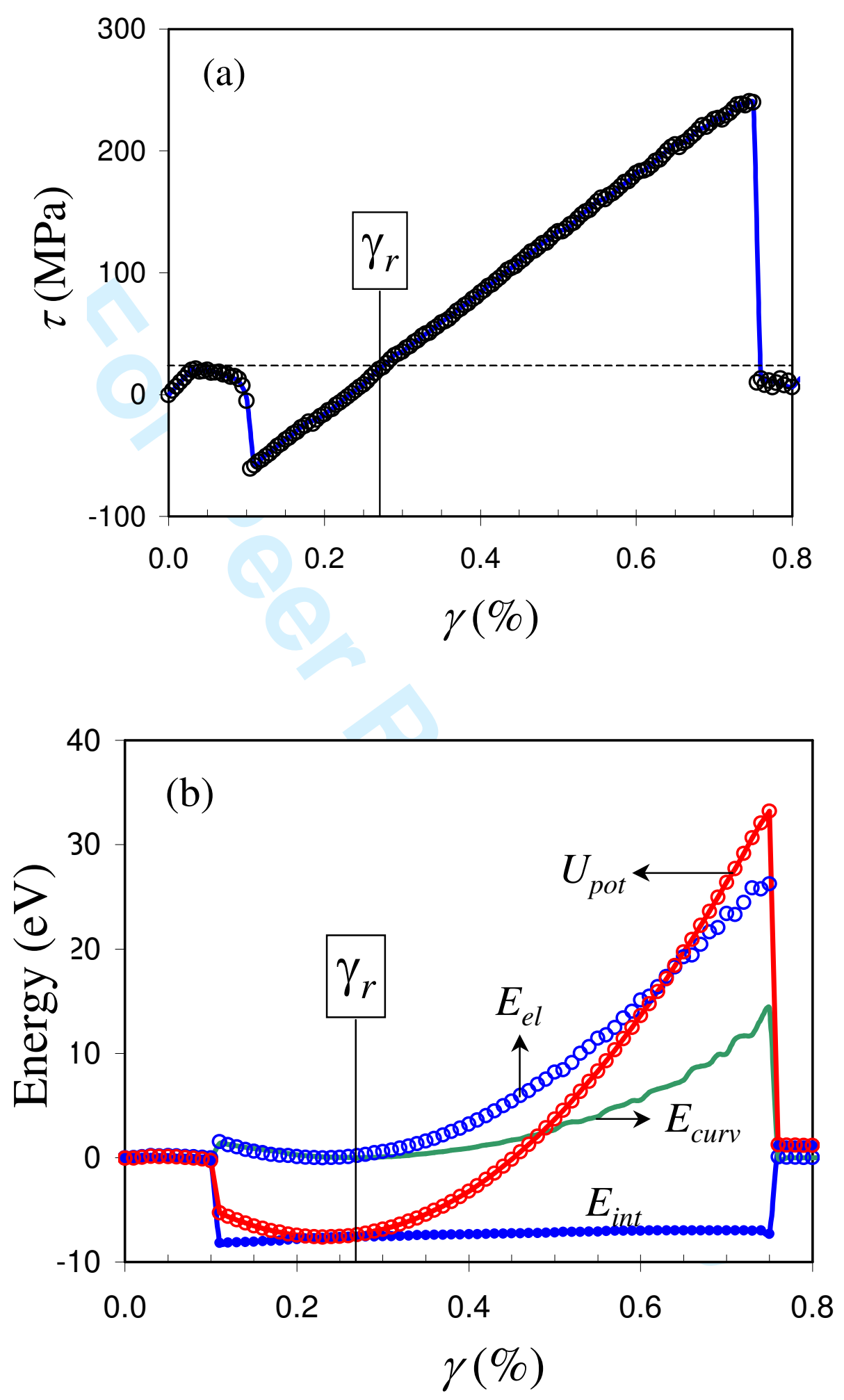

Fig. 1 
(a)

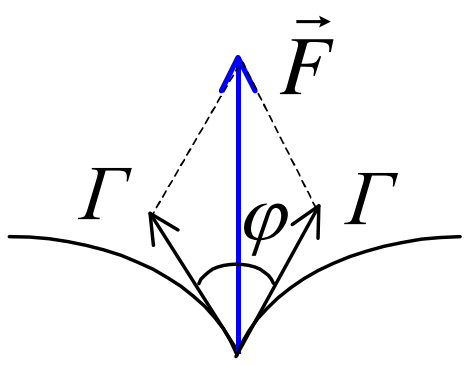

(b) $\tau_{\text {eff }}$

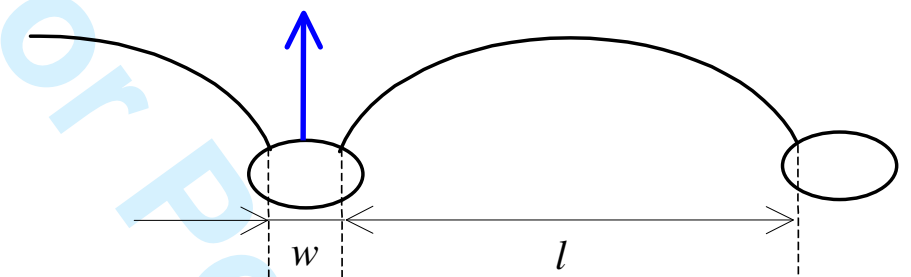

Fig. 2 


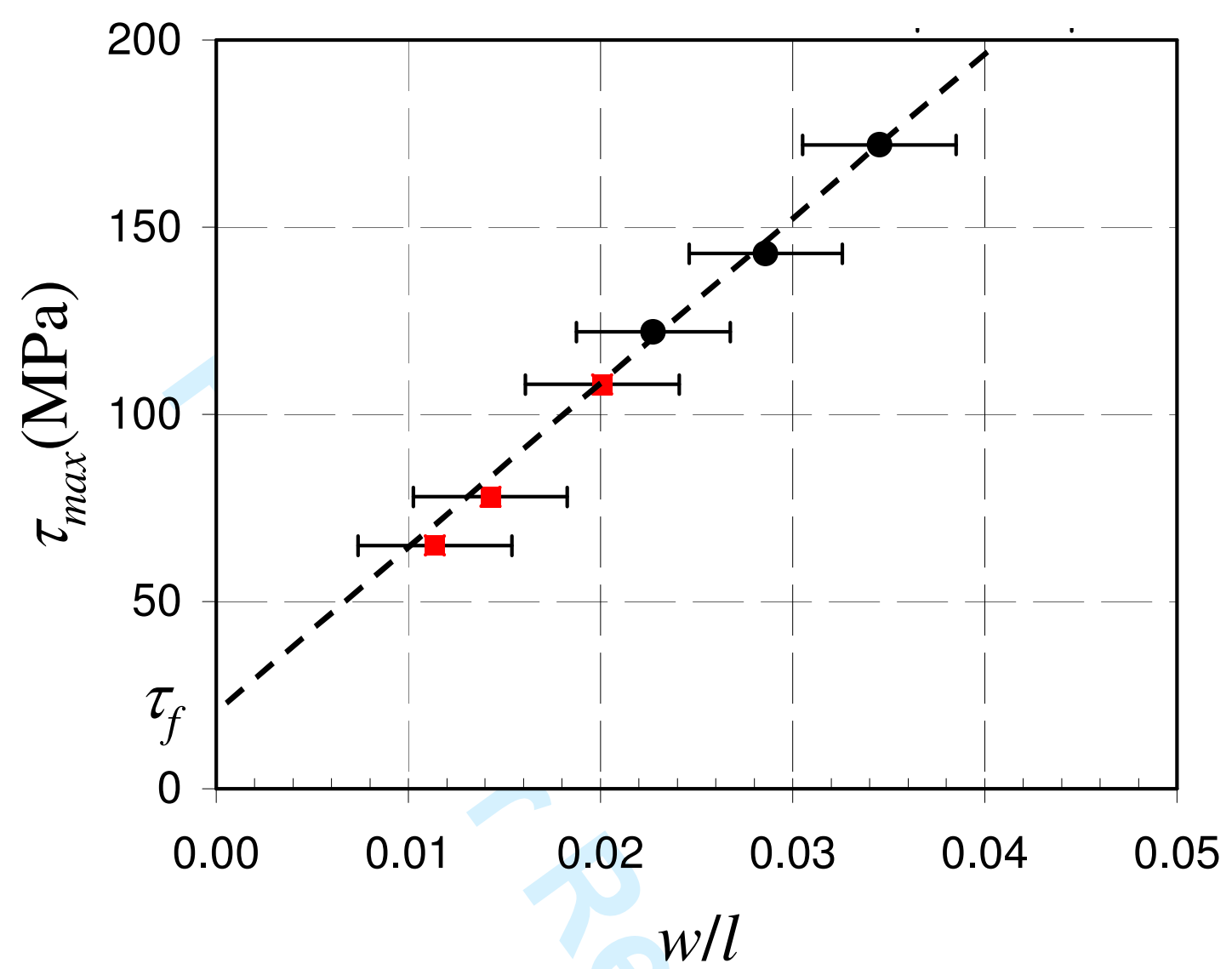

Fig.3 

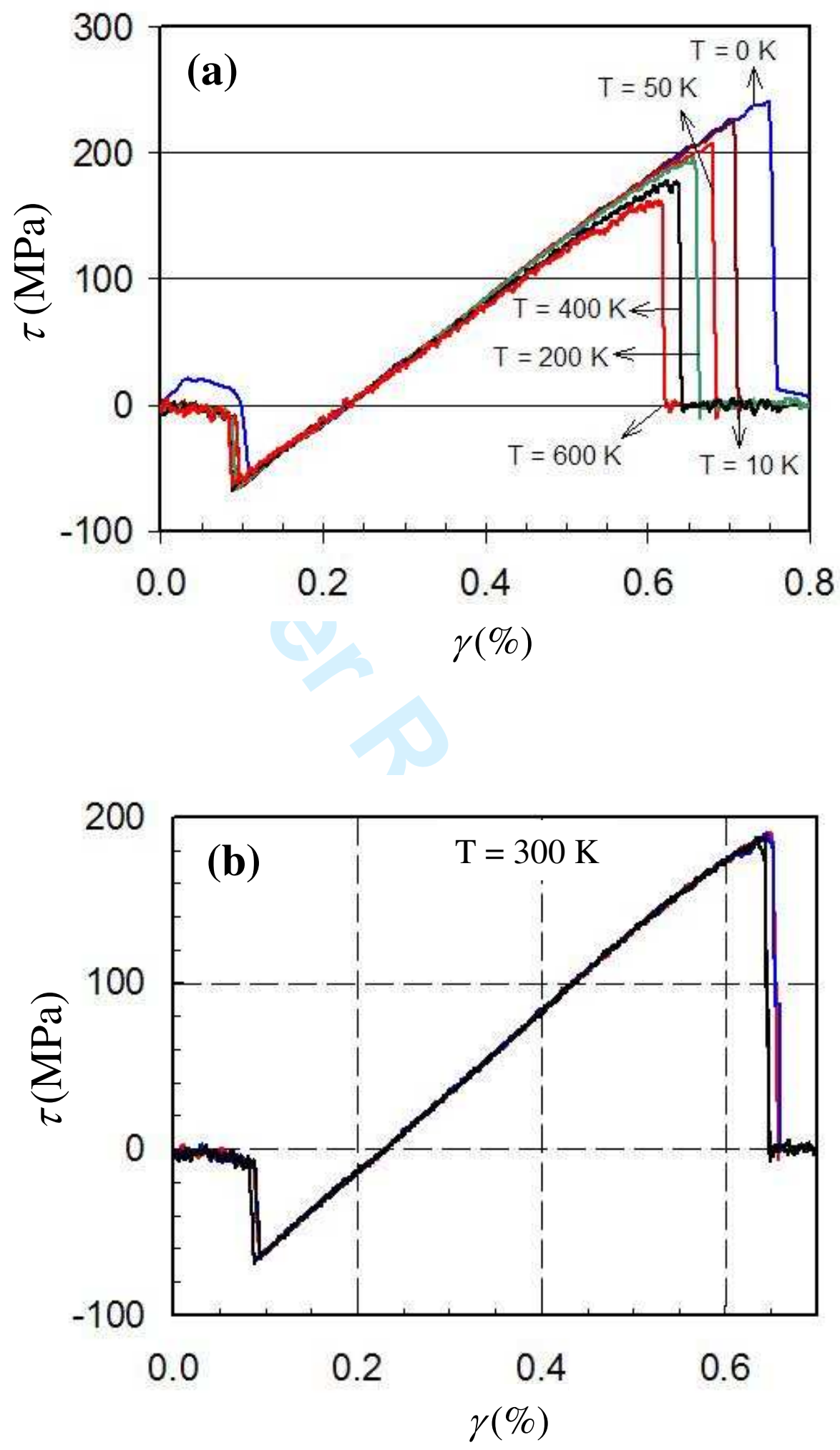

Fig.4 


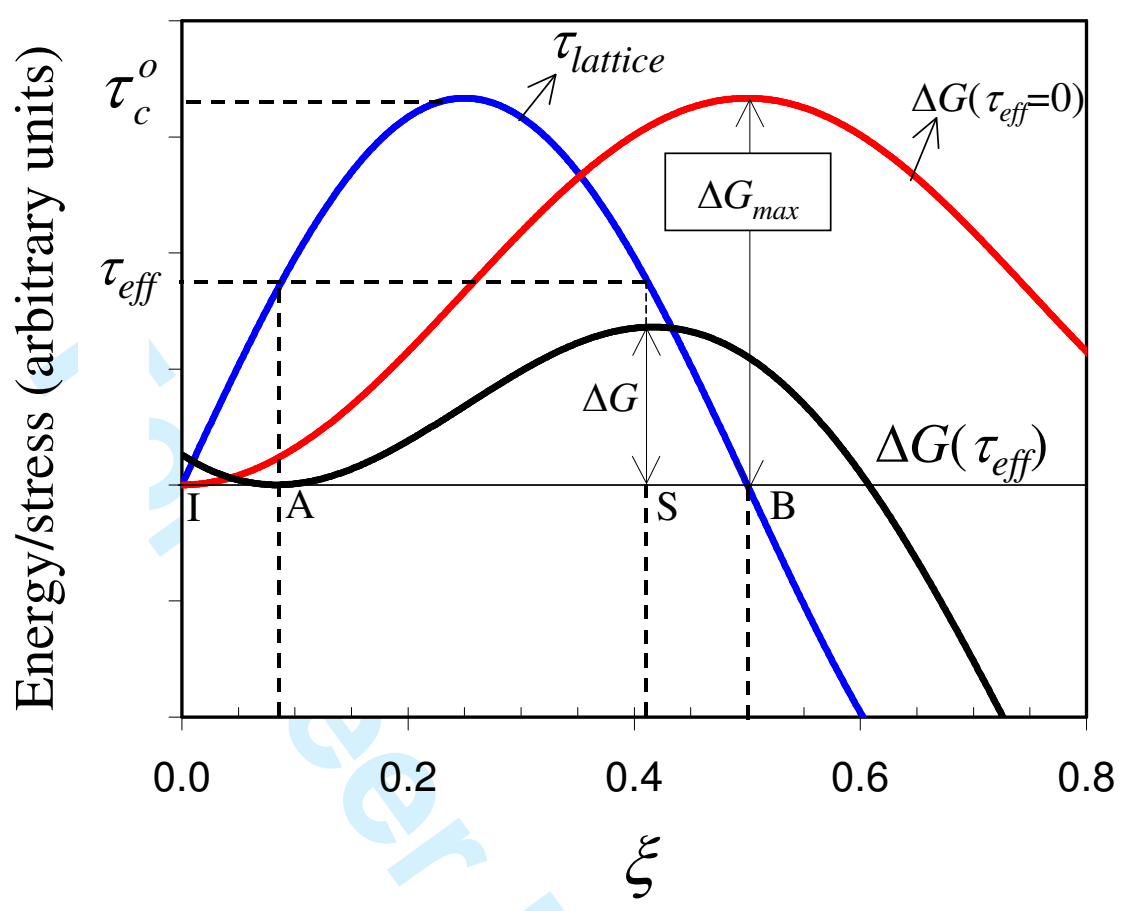

Fig.5 


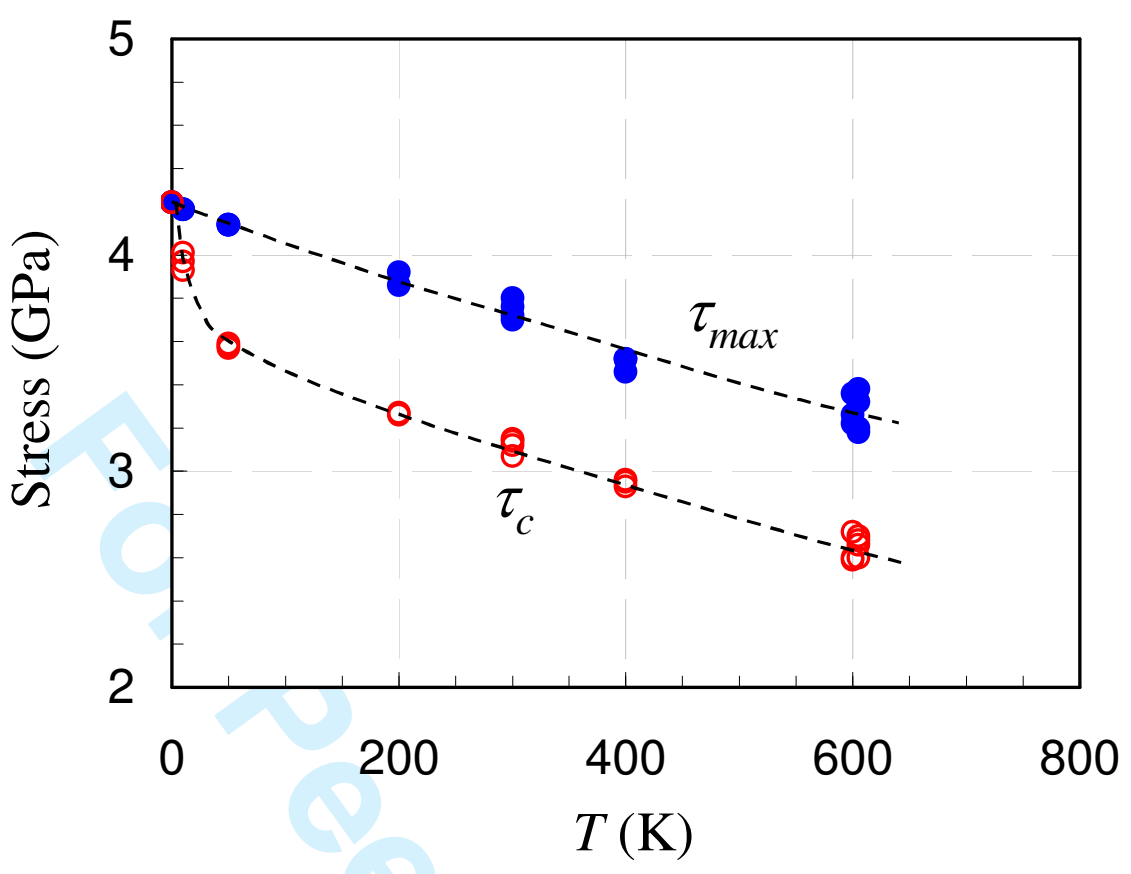

Fig.6 


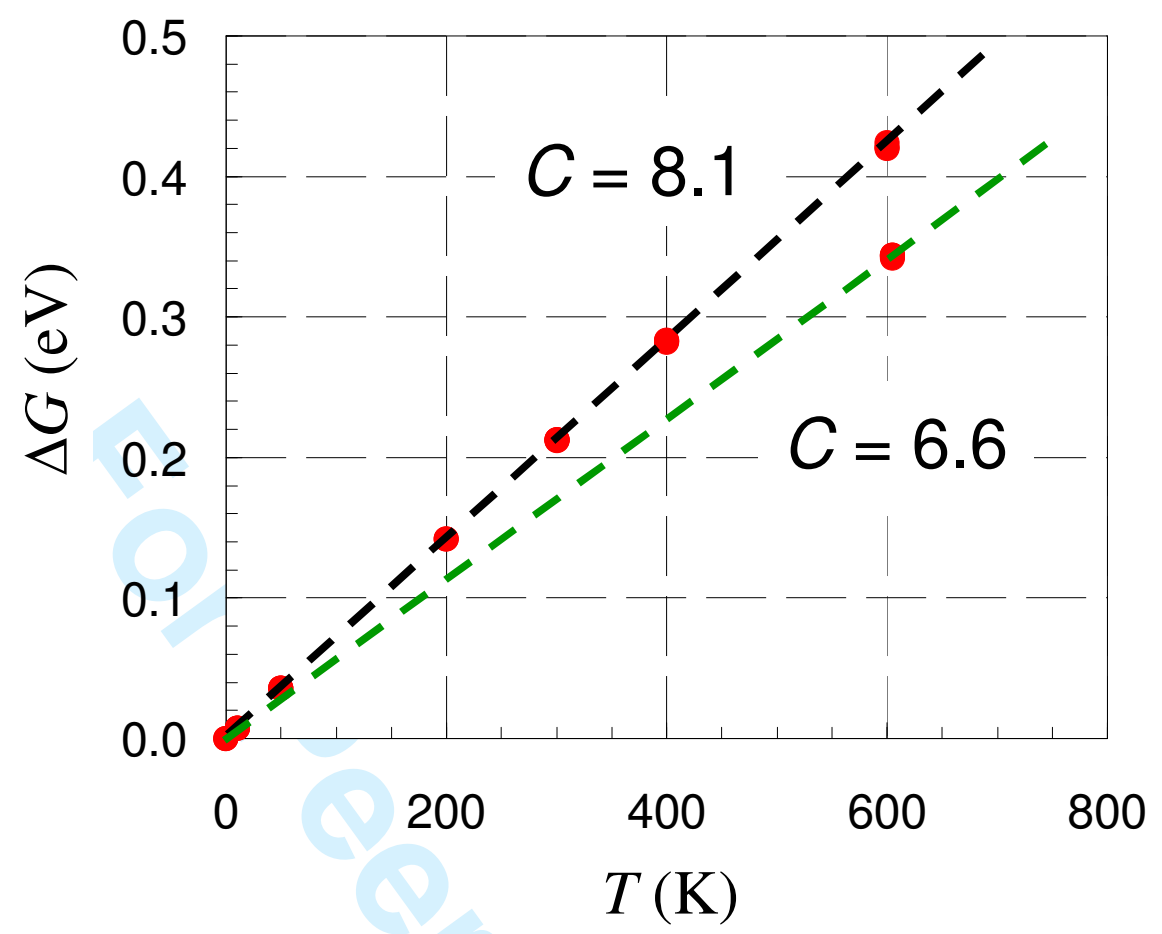

Fig.7 


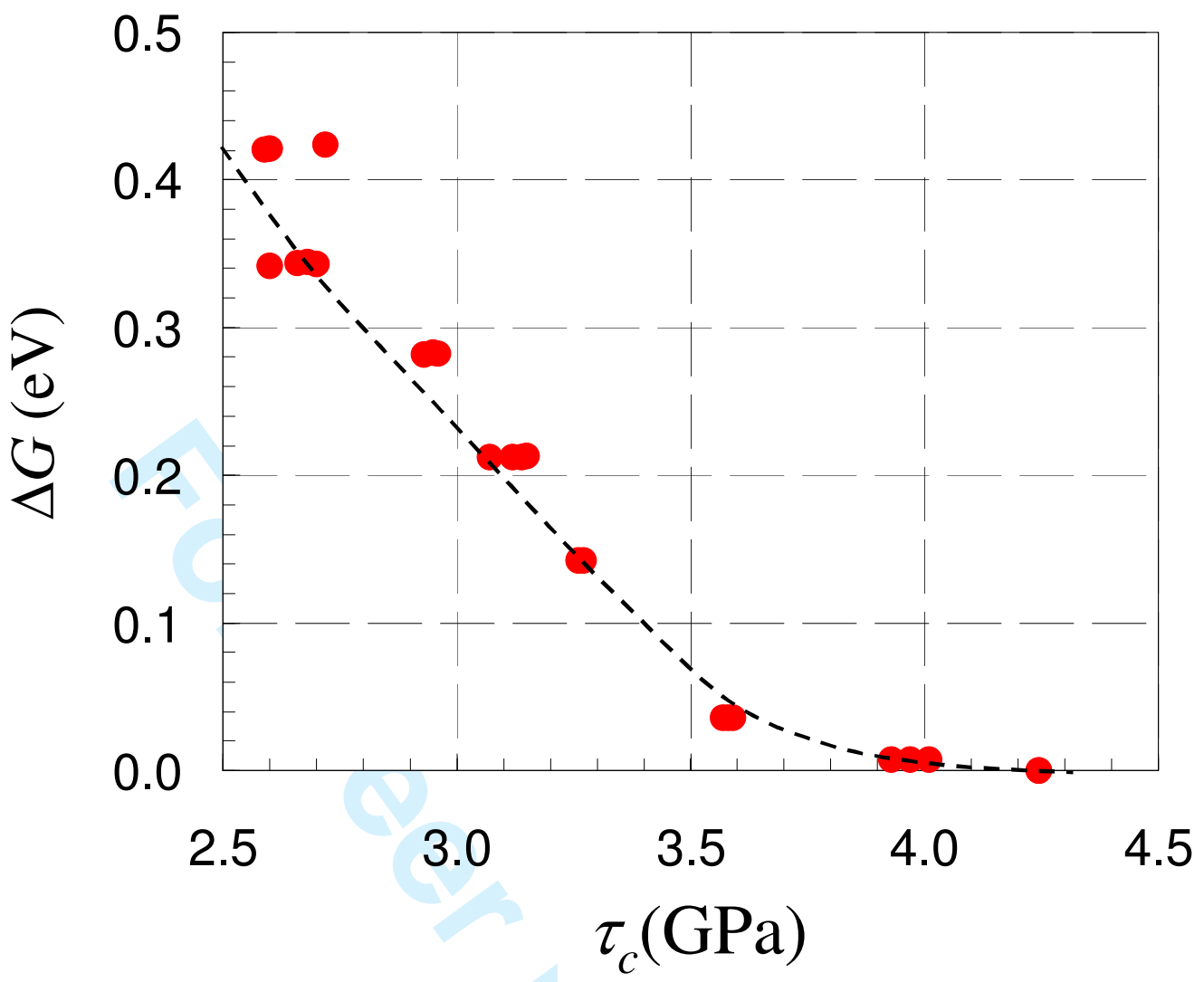

Fig.8 